\title{
Metric of a tidally distorted, nonrotating black hole
}

\author{
Eric Poisson \\ Department of Physics, University of Guelph, Guelph, Ontario, Canada N1G 2W1
}

(Dated: March 8, 2005)

\begin{abstract}
The metric of a tidally distorted, nonrotating black hole is presented in a light-cone coordinate system that penetrates the event horizon and possesses a clear geometrical meaning. The metric is expressed as an expansion in powers of $r / \mathcal{R} \ll 1$, where $r$ is a measure of distance from the black hole and $\mathcal{R}$ is the local radius of curvature of the external spacetime; this is assumed to be much larger than $M$, the mass of the black hole. The metric is calculated up to a remainder of order $(r / \mathcal{R})^{4}$, and it depends on a family of tidal gravitational fields which characterize the hole's local environment. The coordinate system allows an easy identification of the event horizon, and expressions are derived for its surface gravity and the rates at which the tidal interaction transfers mass and angular momentum to the black hole.
\end{abstract}

Introduction. The numerical integration of the Einstein field equations for the simulation of a two-body system of black holes is currently the subject of intense research activity. This work is largely motivated by ongoing attempts to measure gravitational waves, an effort which requires the theoretical input of detailed predictions for the expected waves. The numerical simulations must proceed from initial data that correctly describe a situation of astrophysical interest, a spacelike hypersurface that contains two black holes in bound orbital motion, together with the gravitational radiation that was emitted by the system in the recent past. To find realistic initial data has proved a challenge [1, and it has been suggested 2, 3] that these could be reliably constructed using post-Newtonian (and post-Minkowskian) methods.

While a post-Newtonian metric is adequate to describe the mutual gravity of a weakly-bound two-body system, it fails to describe the strong gravity of each black hole. Building on techniques developed by previous authors [4, 5], Alvi remedied this situation [3] by matching the post-Newtonian metric to two perturbed versions of the Schwarzschild metric (each black hole being tidally distorted by the other hole), in a buffer region in which both approximations are valid. Alvi's metric is ready to use in numerical simulations, and in this Letter we improve on a part of Alvi's construction by providing a more accurate version of the perturbed Schwarzschild metric.

We calculate the metric of a tidally distorted, nonrotating black hole to a level of accuracy that has never been attempted before. The hole is immersed in an external spacetime whose local radius of curvature is $\mathcal{R}$; this is assumed to be much larger than $M$, the mass of the black hole. The tidal distortion is created by the Weyl curvature of the external spacetime, and the Schwarzschild metric acquires a correction that can be expanded in powers of $\mathcal{R}^{-1}$. We calculate all correction terms of order $\mathcal{R}^{-2}$ and $\mathcal{R}^{-3}$; while the former were known previously [6, 7] and used in Alvi's construction [3], the latter are new. The metric is presented in a light-cone coordinate system that penetrates the event horizon and possesses a clear geometrical meaning. These coordinates, which extend over a neighborhood of the black hole that is large relative to $M$ but small relative to $\mathcal{R}$, allow an easy identification of the perturbed event horizon. As an application we calculate the hole's surface gravity and the rates at which the tidal interaction transfers mass and angular momentum to the black hole. Because of space limitations we present our results with very few derivations; a forthcoming paper will supply the missing details.

Advanced coordinates based at a world line. We begin with the construction of a light-cone coordinate system in a neighborhood of a geodesic world line; the only assumption placed on the spacetime is that its Ricci tensor vanishes in the neighborhood. This spacetime will later play the role of the external spacetime, and the world line will later be replaced by the world tube traced by the black hole. The quasi-Cartesian version of the advanced coordinates is denoted $\left(v, x^{a}\right)$, and the quasispherical version $\left(v, r, \theta^{A}\right)$. The null coordinate $v$ labels past light cones that are centered on the world line, $r$ is an areal radius and an affine parameter on each cone's null generators (with $r=0$ on the world line), and the angles $\theta^{A}=(\theta, \phi)(A=2,3)$ are constant on each generator. The coordinates $x^{a}(a=1,2,3)$ are defined by $x^{a}:=r \Omega^{a}\left(\theta^{A}\right)$ with $\Omega^{a}=(\sin \theta \cos \phi, \sin \theta \sin \phi, \cos \theta)$.

Let $\gamma$ denote the geodesic world line, which is parameterized by proper time $\tau$; its normalized tangent vector is $u^{\mu}$. Let $e_{a}^{\mu}(\tau)$ be a triad of orthonormal vectors (all orthogonal to $u^{\mu}$ ) which are parallel transported on the world line. Let $\sigma\left(x, x^{\prime}\right)$ be half the squared geodesic distance between $x$ and $x^{\prime}$, and let $\sigma_{\alpha^{\prime}}\left(x, x^{\prime}\right)$ denote its gradient with respect to $x^{\prime}$. The advanced coordinates are defined as follows. Let $x^{\prime}$ be the point on the world line at which $\tau$ takes the value $v$. The set of events $x$ such that $\sigma\left(x, x^{\prime}\right)=0$ and $x$ is in the past of $x^{\prime}$ defines the past light cone of $x^{\prime}$. We assign the advanced-time coordinate $v$ to all these events. The spatial coordinates of a point $x$ on the cone are then $x^{a}=-\delta^{a b} e_{b}^{\alpha^{\prime}}(v) \sigma_{\alpha^{\prime}}\left(x, x^{\prime}\right)$. We introduce $r:=\left(\delta_{a b} x^{a} x^{b}\right)^{1 / 2}$, which can be shown to be an affine parameter on the null generators of the cone $v=$ constant. We also introduce $\Omega^{a}:=x^{a} / r$, which may be parameterized by the two angles $\theta^{A}$. It can further be 
TABLE I: Irreducible tidal fields. Each field is identified with a sans-serif superscript that specifies its multipole content. A field labeled with a "q" is a quadrupole field, and one labeled with an "o" is an octupole field. The vector and tensor fields are all orthogonal to $\Omega^{a}$, and all tensor fields are symmetric and tracefree.

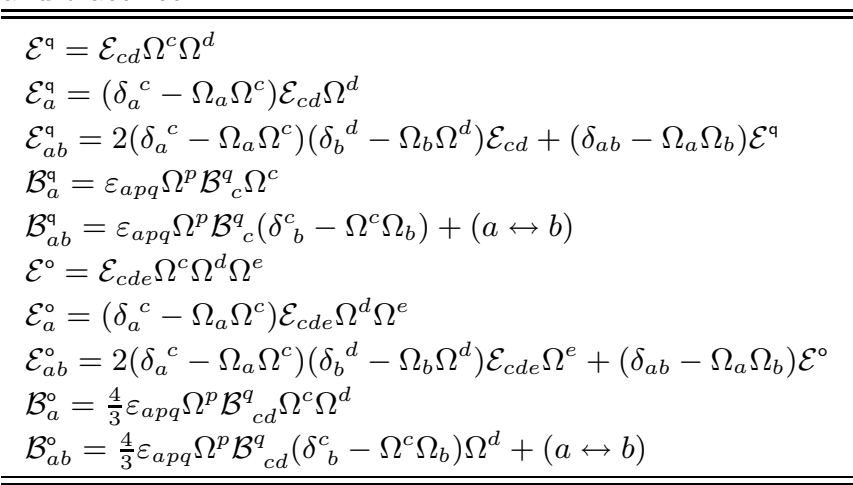

shown that $\theta^{A}$ are constant on the null generators.

Tensors on $\gamma$ can be decomposed in the tetrad $\left(u^{\mu}, e_{a}^{\mu}\right)$. For example, $C_{a 0 b 0}:=C_{\mu \lambda \nu \rho} e_{a}^{\mu} u^{\lambda} e_{b}^{\nu} u^{\rho}$ and $C_{a b c 0}:=$ $C_{\mu \lambda \nu \rho} e_{a}^{\mu} e_{b}^{\lambda} e_{c}^{\nu} u^{\rho}$ are frame components of the Weyl tensor evaluated on $\gamma$. Similarly, $\dot{C}_{a 0 b 0}:=C_{\mu \lambda \nu \rho ; \omega} e_{a}^{\mu} u^{\lambda} e_{b}^{\nu} u^{\rho} u^{\omega}$ and $C_{a 0 b 0 \mid c}:=C_{\mu \lambda \nu \rho ; \omega} e_{a}^{\mu} u^{\lambda} e_{b}^{\nu} u^{\rho} e_{c}^{\omega}$ are frame components of the covariantly differentiated Weyl tensor. The 10 independent components of the Weyl tensor are conveniently related to two symmetric-tracefree (STF) frame tensors [4], $\mathcal{E}_{a b}:=C_{a 0 b 0}$ and $\mathcal{B}_{a b}:=\frac{1}{2} \varepsilon_{a}^{p q} C_{p q b 0}$, where $\varepsilon_{a b c}$ is the permutation symbol (frame indices are freely manipulated with $\delta_{a b}$ ). The components of the differentiated Weyl tensor can also be expressed in terms of STF frame tensors; these include $\dot{\mathcal{E}}_{a b}$ and $\dot{\mathcal{B}}_{a b}$ as well as $\mathcal{E}_{a b c}:=\frac{1}{3}\left(C_{a 0 b 0 \mid c}+C_{c 0 a 0 \mid b}+C_{b 0 c 0 \mid a}\right)$ and $\mathcal{B}_{a b c}:=$ $\frac{1}{8}\left(\varepsilon_{a}^{p q} C_{p q b 0 \mid c}+\varepsilon_{c}^{p q} C_{p q a 0 \mid b}+\varepsilon_{b}^{p q} C_{p q c 0 \mid a}\right)$.

These frame tensors allow us to define length scales 4. that characterize the tidal gravitational field acting at $\gamma$. Let $\mathcal{R}$ be the local radius of curvature, defined such that $\mathcal{E}_{a b} \sim \mathcal{B}_{a b} \sim \mathcal{R}^{-2}$. Let $\mathcal{L}$ be a scale of spatial inhomogeneity, defined such that $\mathcal{E}_{a b c} \sim \mathcal{B}_{a b c} \sim \mathcal{R}^{-2} \mathcal{L}^{-1}$. And let $\mathcal{T}$ be a time scale associated with changes in the tidal field, defined such that $\dot{\mathcal{E}}_{a b} \sim \dot{\mathcal{B}}_{a b} \sim \mathcal{R}^{-2} \mathcal{T}^{-1}$. In the sequel we assume, for simplicity, that $\mathcal{R} \sim \mathcal{L} \sim \mathcal{T}$ and use $\mathcal{R}$ as the fundamental length scale associated with the tidal field.

The procedure to obtain the spacetime's metric in the coordinates $\left(v, x^{a}\right)$ or $\left(v, r, \theta^{A}\right)$ is detailed in Ref. [7]. The metric is expressed as an expansion in powers of $r / \mathcal{R}$, which is assumed to be small; the coordinate domain is therefore limited to a neighborhood of $\gamma$. The appearance of the metric is simplified by forming irreducible combinations involving the tidal fields $\mathcal{E}_{a b}(v), \mathcal{E}_{a b c}(v), \mathcal{B}_{a b}(v)$, $\mathcal{B}_{a b c}(v)$, the unit frame vector $\Omega^{a}=x^{a} / r$, the permutation symbol $\varepsilon_{a b c}$, and the frame metric $\delta_{a b}$. These combinations are displayed in Table I.
The quasi-Cartesian form of the metric is

$$
\begin{aligned}
g_{v v}= & -1-r^{2} \mathcal{E}^{q}+\frac{1}{3} r^{3} \dot{\mathcal{E}}^{q}-\frac{1}{3} r^{3} \mathcal{E}^{\circ}+O(4) \\
g_{v a}= & \Omega_{a}-\frac{2}{3} r^{2}\left(\mathcal{E}_{a}^{a}-\mathcal{B}_{a}^{q}\right)+\frac{1}{3} r^{3}\left(\dot{\mathcal{E}}_{a}^{q}-\dot{\mathcal{B}}_{a}^{q}\right) \\
& -\frac{1}{4} r^{3}\left(\mathcal{E}_{a}^{\circ}-\mathcal{B}_{a}^{\circ}\right)+O(4) \\
g_{a b}= & \delta_{a b}-\Omega_{a} \Omega_{b}-\frac{1}{3} r^{2}\left(\mathcal{E}_{a b}^{q}-\mathcal{B}_{a b}^{q}\right)+\frac{5}{18} r^{3}\left(\dot{\mathcal{E}}_{a b}^{q}-\dot{\mathcal{B}}_{a b}^{q}\right) \\
& -\frac{1}{6} r^{3}\left(\mathcal{E}_{a b}^{\circ}-\mathcal{B}_{a b}^{\circ}\right)+O(4)
\end{aligned}
$$

where $O(4)$ stands for "a remainder that scales as $\mathcal{R}^{-4}$." The quasi-spherical form of the metric is obtained by performing the coordinate transformation $x^{a}=r \Omega^{a}\left(\theta^{A}\right)$. Its nonvanishing components are $g_{v v}$ given by Eq. (1), $g_{v r}=1$, and

$$
\begin{aligned}
g_{v A}= & -\frac{2}{3} r^{3}\left(\mathcal{E}_{A}^{\mathrm{q}}-\mathcal{B}_{A}^{\mathrm{q}}\right)+\frac{1}{3} r^{4}\left(\dot{\mathcal{E}}_{A}^{\mathrm{q}}-\dot{\mathcal{B}}_{A}^{\mathrm{q}}\right) \\
& -\frac{1}{4} r^{4}\left(\mathcal{E}_{A}^{\circ}-\mathcal{B}_{A}^{\circ}\right)+O(4), \\
g_{A B}= & r^{2} \Omega_{A B}-\frac{1}{3} r^{4}\left(\mathcal{E}_{A B}^{\mathrm{q}}-\mathcal{B}_{A B}^{\mathrm{q}}\right)+\frac{5}{18} r^{5}\left(\dot{\mathcal{E}}_{A B}^{\mathrm{q}}-\dot{\mathcal{B}}_{A B}^{\mathrm{q}}\right) \\
& -\frac{1}{6} r^{5}\left(\mathcal{E}_{A B}^{\circ}-\mathcal{B}_{A B}^{\circ}\right)+O(4) .
\end{aligned}
$$

Here $\Omega_{A B}:=\delta_{a b} \Omega_{A}^{a} \Omega_{B}^{b}=\operatorname{diag}\left(1, \sin ^{2} \theta\right), \mathcal{E}_{A}^{q}:=\mathcal{E}_{a}^{a} \Omega_{A}^{a}$, $\mathcal{E}_{A B}^{q}:=\mathcal{E}_{a b}^{q} \Omega_{A}^{a} \Omega_{B}^{b}$, and so on, with $\Omega_{A}^{a}:=\partial \Omega^{a} / \partial \theta^{A}$. The irreducible tidal fields can all be expanded in spherical harmonics, and this decomposition is presented in Table II. According to Eqs. (11), (4), and (5), $\sqrt{-g}=r^{2} \sin \theta[1+$ $O(4)$ ], which implies that surfaces of constant $v$ and $r$ have an area of $4 \pi r^{2}[1+O(4)]$.

Metric of a tidally distorted black hole. The metric of an isolated, nonrotating black hole of mass $M$ can be put in the form

$$
g_{\alpha \beta}^{0} d x^{\alpha} d x^{\beta}=-f d v^{2}+2 d v d r+r^{2} \Omega_{A B} d \theta^{A} d \theta^{B},
$$

where $f:=1-2 M / r$ and $\Omega_{A B} d \theta^{A} d \theta^{B}=d \theta^{2}+\sin ^{2} \theta d \phi^{2}$. The coordinates $\left(v, r, \theta^{A}\right)$ have the same meaning as in the preceding section: $v$ labels past light cones, $r$ is an areal radius and an affine parameter on each cone's null generators, and $\theta^{A}$ are constant on each generator. The metric of Eq. (6) approaches the Minkowski metric in the asymptotic regime $r \gg 2 M$.

A tidally distorted black hole is described by a metric that is a perturbed version of Eq. (6). In the asymptotic regime $r \gg 2 M$ (keeping $r \ll \mathcal{R}$, which implies that $M$ must be much smaller than $\mathcal{R}$ ) this metric must approach the metric of Eqs. (11), (4), and (5) instead of the Minkowski metric. This observation motivates the following ansatz for the perturbed metric:

$$
g_{v v}=-f-r^{2} e_{1} \mathcal{E}^{a}+\frac{1}{3} r^{3} e_{2} \dot{\mathcal{E}}^{\natural}-\frac{1}{3} r^{3} e_{3} \mathcal{E}^{\circ}+O(4),
$$


TABLE II: Spherical-harmonic decomposition of the irreducible tidal fields. The first column lists $Y^{l \mathrm{~m}}\left(\theta^{A}\right)$, the real-valued spherical-harmonic functions that are used in the decomposition. The abstract index $\mathrm{m}$ describes the dependence of these functions on $\phi$; for example $Y^{3,2 s}$ is proportional to $\sin 2 \phi$. The second and third columns define $\mathcal{E}_{\mathrm{m}}^{\mathrm{q}}(v), \mathcal{E}_{\mathrm{m}}^{\circ}(v), \mathcal{B}_{\mathrm{m}}^{\mathrm{q}}(v), \mathcal{B}_{\mathrm{m}}^{\circ}(v)$, the harmonic components of the tidal fields, in terms of their frame components $\mathcal{E}_{a b}(v), \mathcal{E}_{a b c}(v), \mathcal{B}_{a b}(v), \mathcal{B}_{a b c}(v)$. The fourth column displays the spherical-harmonic decompositions of the quadrupole and octupole tidal fields. The vectorial spherical harmonics are $Y_{A}^{l \mathrm{~m}}:=D_{A} Y^{l \mathrm{~m}}$ and $X_{A}^{l \mathrm{~m}}:=-\varepsilon_{A}^{B} D_{B} Y^{l \mathrm{~m}}$, where $D_{A}$ is the covariant derivative operator on the unit two-sphere $\left(D_{A} \Omega_{B C}=0\right)$ and $\varepsilon_{A B}$ is the Levi-Civita tensor $\left(\varepsilon_{\theta \phi}=\sin \theta\right.$; indices raised with $\Omega^{A B}$, the inverse two-sphere metric). The tensorial spherical harmonics are $Y_{A B}^{l \mathrm{~m}}:=\left[D_{A} D_{B}+\frac{1}{2} l(l+1) \Omega_{A B}\right] Y^{l \mathrm{~m}}$ and $X_{A B}^{l \mathrm{~m}}=-\frac{1}{2}\left(\varepsilon_{A}^{C} D_{B}+\varepsilon_{B}^{C} D_{A}\right) D_{C} Y^{l \mathrm{~m}}$.

\begin{tabular}{llll}
\hline \hline$Y^{2,0}=-\left(3 \cos ^{2} \theta-1\right)$ & $\mathcal{E}_{0}^{\mathrm{q}}=\frac{1}{2}\left(\mathcal{E}_{11}+\mathcal{E}_{22}\right)$ & $\mathcal{B}_{0}^{\mathrm{q}}=\frac{1}{2}\left(\mathcal{B}_{11}+\mathcal{B}_{22}\right)$ & $\mathcal{E}^{\mathrm{q}}=\sum_{\mathrm{m}} \mathcal{E}_{\mathrm{m}}^{\mathrm{q}} Y^{2 \mathrm{~m}}$ \\
$Y^{2,1 c}=2 \sin \theta \cos \theta \cos \phi$ & $\mathcal{E}_{1 c}^{\mathrm{q}}=\mathcal{E}_{13}$ & $\mathcal{B}_{1 c}^{\mathrm{q}}=\mathcal{B}_{13}$ & $\mathcal{E}_{A}^{\mathrm{q}}=\frac{1}{2} \sum_{\mathrm{m}} \mathcal{E}_{\mathrm{m}}^{\mathrm{q}} Y_{A}^{2 \mathrm{~m}}$ \\
$Y^{2,1 s}=2 \sin \theta \cos \theta \sin \phi$ & $\mathcal{E}_{1 s}^{\mathrm{q}}=\mathcal{E}_{23}$ & $\mathcal{B}_{1 s}^{\mathrm{q}}=\mathcal{B}_{23}$ & $\mathcal{E}_{A B}^{\mathrm{q}}=\sum_{\mathrm{m}} \mathcal{E}_{\mathrm{m}}^{\mathrm{q}} Y_{A B}^{2 \mathrm{~m}}$ \\
$Y^{2,2 c}=\sin ^{2} \theta \cos 2 \phi$ & $\mathcal{E}_{2 c}^{\mathrm{q}}=\frac{1}{2}\left(\mathcal{E}_{11}-\mathcal{E}_{22}\right)$ & $\mathcal{B}_{2 c}^{\mathrm{q}}=\frac{1}{2}\left(\mathcal{B}_{11}-\mathcal{B}_{22}\right)$ & $\mathcal{B}_{A}^{\mathrm{q}}=\frac{1}{2} \sum_{\mathrm{m}} \mathcal{B}_{\mathrm{m}}^{\mathrm{q}} X_{A}^{2 \mathrm{~m}}$ \\
$Y^{2,2 s}=\sin ^{2} \theta \sin 2 \phi$ & $\mathcal{E}_{2 s}^{\mathrm{q}}=\mathcal{E}_{12}$ & $\mathcal{B}_{2 s}^{\mathrm{q}}=\mathcal{B}_{12}$ & $\mathcal{B}_{A B}^{\mathrm{q}}=\sum_{\mathrm{m}} \mathcal{B}_{\mathrm{m}}^{\mathrm{q}} X_{A B}^{2 \mathrm{~m}}$ \\
$Y^{3,0}=-\left(5 \cos ^{3} \theta-3 \cos \theta\right)$ & $\mathcal{E}_{0}^{\circ}=\frac{1}{2}\left(\mathcal{E}_{113}+\mathcal{E}_{223}\right)$ & $\mathcal{B}_{0}^{\circ}=\frac{2}{3}\left(\mathcal{B}_{113}+\mathcal{B}_{223}\right)$ & \\
$Y^{3,1 c}=-\frac{3}{2} \sin \theta\left(5 \cos ^{2} \theta-1\right) \cos \phi$ & $\mathcal{E}_{1 c}^{\circ}=\frac{1}{2}\left(\mathcal{E}_{111}+\mathcal{E}_{122}\right)$ & $\mathcal{B}_{1 c}^{\circ}=\frac{2}{3}\left(\mathcal{B}_{111}+\mathcal{B}_{122}\right)$ & $\mathcal{E}^{\circ}=\sum_{\mathrm{m}} \mathcal{E}_{\mathrm{m}}^{\circ} Y^{3 \mathrm{~m}}$ \\
$Y^{3,1 s}=-\frac{3}{2} \sin \theta\left(5 \cos ^{2} \theta-1\right) \sin \phi$ & $\mathcal{E}_{1 s}^{\circ}=\frac{1}{2}\left(\mathcal{E}_{112}+\mathcal{E}_{222}\right)$ & $\mathcal{B}_{1 s}^{\circ}=\frac{2}{3}\left(\mathcal{B}_{112}+\mathcal{B}_{222}\right)$ & $\mathcal{E}_{A}^{\circ}=\frac{1}{3} \sum_{\mathrm{m}} \mathcal{E}_{\mathrm{m}}^{\circ} Y_{A}^{3 \mathrm{~m}}$ \\
$Y^{3,2 c}=3 \sin ^{2} \theta \cos \theta \cos 2 \phi$ & $\mathcal{E}_{2 c}^{\circ}=\frac{1}{2}\left(\mathcal{E}_{113}-\mathcal{E}_{223}\right)$ & $\mathcal{B}_{2 c}^{\circ}=\frac{2}{3}\left(\mathcal{B}_{113}-\mathcal{B}_{223}\right)$ & $\mathcal{E}_{A B}^{\circ}=\frac{1}{3} \sum_{\mathrm{m}} \mathcal{E}_{\mathrm{m}}^{\circ} Y_{A B}^{3 \mathrm{~m}}$ \\
$Y^{3,2 s}=3 \sin ^{2} \theta \cos \theta \sin 2 \phi$ & $\mathcal{E}_{2 s}^{\circ}=\mathcal{E}_{123}$ & $\mathcal{B}_{2 s}^{\circ}=\frac{4}{3} \mathcal{B}_{123}$ & $\mathcal{B}_{A}^{\circ}=\frac{1}{3} \sum_{\mathrm{m}} \mathcal{B}_{\mathrm{m}}^{\circ} X_{A}^{3 \mathrm{~m}}$ \\
$Y^{3,3 c}=\sin ^{3} \theta \cos 3 \phi$ & $\mathcal{E}_{3 c}^{\circ}=\frac{1}{4}\left(\mathcal{E}_{111}-3 \mathcal{E}_{122}\right)$ & $\mathcal{B}_{3 c}^{\circ}=\frac{1}{3}\left(\mathcal{B}_{111}-3 \mathcal{B}_{122}\right)$ & $\mathcal{B}_{A B}^{\circ}=\frac{1}{3} \sum_{\mathrm{m}} \mathcal{B}_{\mathrm{m}}^{\circ} X_{A B}^{3 \mathrm{~m}}$ \\
$Y^{3,3 s}=\sin ^{3} \theta \sin 3 \phi$ & $\mathcal{E}_{3 s}^{\circ}=\frac{1}{4}\left(3 \mathcal{E}_{112}-\mathcal{E}_{222}\right)$ & $\mathcal{B}_{3 s}^{\circ}=\frac{1}{3}\left(3 \mathcal{B}_{112}-\mathcal{B}_{222}\right)$ & \\
\hline \hline
\end{tabular}

$$
\begin{aligned}
g_{v r}= & 1 \\
g_{v A}= & -\frac{2}{3} r^{3}\left(e_{4} \mathcal{E}_{A}^{\mathrm{q}}-b_{4} \mathcal{B}_{A}^{\mathrm{q}}\right)+\frac{1}{3} r^{4}\left(e_{5} \dot{\mathcal{E}}_{A}^{\mathrm{q}}-b_{5} \dot{\mathcal{B}}_{A}^{\mathrm{q}}\right) \\
& -\frac{1}{4} r^{4}\left(e_{6} \mathcal{E}_{A}^{\circ}-b_{6} \mathcal{B}_{A}^{\circ}\right)+O(4), \\
g_{A B}= & r^{2} \Omega_{A B}-\frac{1}{3} r^{4}\left(e_{7} \mathcal{E}_{A B}^{\mathrm{q}}-b_{7} \mathcal{B}_{A B}^{\mathrm{q}}\right) \\
& +\frac{5}{18} r^{5}\left(e_{8} \dot{\mathcal{E}}_{A B}^{\mathrm{q}}-b_{8} \dot{\mathcal{B}}_{A B}^{\mathrm{q}}\right) \\
& -\frac{1}{6} r^{5}\left(e_{9} \mathcal{E}_{A B}^{\circ}-b_{9} \mathcal{B}_{A B}^{\circ}\right)+O(4) .
\end{aligned}
$$

The radial functions $e_{n}(r), b_{n}(r)$ must be determined by integrating the Einstein field equations; they are independent of $\mathcal{R}$, they are required to be well-behaved at $r=2 M$, and they must approach unity when $r \gg 2 M$. The perturbed metric of Eqs. (7)-(10) is expressed in a gauge that preserves the geometrical meaning of the coordinates $\left(v, r, \theta^{A}\right)$; this gauge is both physically meaningful and technically simple. The gauge freedom is not exhausted by adopting the ansatz of Eqs. (7)-(10); refinements can be made when solving the field equations.

The metric of Eqs. (7)-(10) is used to compute the Einstein tensor, which is then expanded in powers of $\mathcal{R}^{-1}$. There is no contribution at orders $\mathcal{R}^{0}$ and $\mathcal{R}^{-1}$, but we examine carefully the contributions at orders $\mathcal{R}^{-2}$ and $\mathcal{R}^{-3}$. At order $\mathcal{R}^{-2}$ the Einstein tensor decouples into spherical-harmonic components that can be handled separately, and it decouples further into even-parity terms proportional to $\mathcal{E}_{\mathrm{m}}^{\mathrm{q}}$ and odd-parity terms proportional to $\mathcal{B}_{\mathrm{m}}^{\mathrm{q}}$. The even-parity sector gives rise to a coupled set of ordinary differential equations for $\left\{e_{1}, e_{4}, e_{7}\right\}$, while the odd-parity sector determines $\left\{b_{4}, b_{7}\right\}$. General solutions are easily found, and they are constrained by demanding that the radial functions be well-behaved at $r=2 M$ and approach unity as $r \rightarrow \infty$. This leaves a number of integration constants undetermined, and those can be freely assigned; this represents a refinement of the gauge, and we adjust the constants so that a maximum number of radial functions vanish at $r=2 M$.

This procedure can be completed at order $\mathcal{R}^{-3}$. Here the octupole terms (proportional to $\mathcal{E}_{\mathrm{m}}^{\circ}$ and $\mathcal{B}_{\mathrm{m}}^{\circ}$ ) decouple from the quadrupole terms (proportional to $\dot{\mathcal{E}}_{\mathrm{m}}^{\mathrm{q}}$ and $\dot{\mathcal{B}}_{\mathrm{m}}^{\mathrm{q}}$ ), and as before the even-parity terms decouple from the odd-parity terms. We thus obtain coupled sets of equations for $\left\{e_{2}, e_{5}, e_{8}\right\},\left\{b_{5}, b_{8}\right\},\left\{e_{3}, e_{6}, e_{9}\right\}$, and $\left\{b_{6}, b_{9}\right\}$. These are integrated using the same techniques as for the $\mathcal{R}^{-2}$ part of the metric. The radial functions are listed in Table III.

The quasi-Cartesian form of the perturbed metric is obtained by inserting $e_{n}$ and $b_{n}$ at appropriate places in Eqs. (11)-(3); for example, the coefficient of $\mathcal{E}_{a}^{q}$ in $g_{v a}$ becomes $-\frac{2}{3} r^{2} e_{4}$. The complete determination of the metric requires the specification of the tidal fields $\mathcal{E}_{a b}(v), \mathcal{E}_{a b c}(v), \mathcal{B}_{a b}(v)$, and $\mathcal{B}_{a b c}(v)$. These characterize the black hole's local environment, and they are determined by matching the hole's local metric to a metric that describes a larger portion of the spacetime $[4,5]$. For example, the global metric could be the post-Newtonian metric of a two-body system, and Alvi 3] has shown how to carry out the matching procedure in this context.

The perturbed horizon. To locate the event horizon of the tidally distorted black hole we note first that the vector $r_{\alpha}:=\partial_{\alpha} r$ is normal to any surface of constant $r$, and that it is null when $r=2 M: g^{\alpha \beta} r_{\alpha} r_{\beta}=O(4)$. The gener- 
TABLE III: Radial functions that appear in the metrics of Eqs. (7)-110), expressed in terms of $x:=r /(2 M)$ and $f:=$ $1-2 M / r$. At $r=2 M$ we have $e_{7}=\frac{1}{2}, e_{9}=\frac{1}{10}, b_{7}=-\frac{1}{2}$, and $b_{9}=-\frac{1}{10}$, with all other functions vanishing.

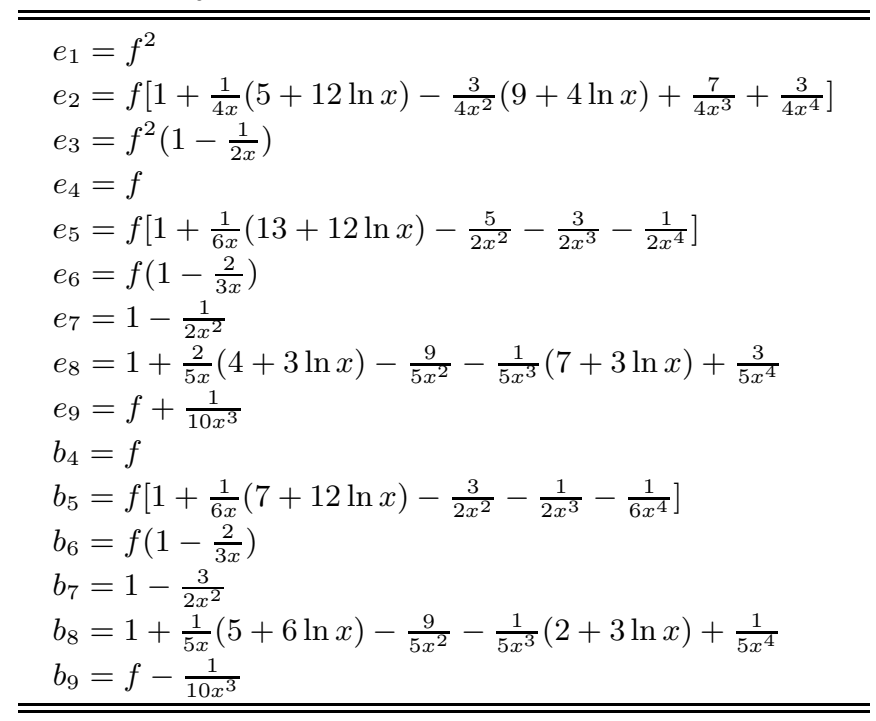

ators of this null surface have $r^{\alpha}$ as their tangent vector, and a simple calculation reveals that $r^{\alpha}=t^{\alpha}+O(4)$, where $t^{\alpha}\left(\partial / \partial x^{\alpha}\right):=\partial / \partial v$. The generators are therefore parameterized by $v$ and they move with constant values of $\theta^{A}$ (up to corrections of order $\mathcal{R}^{-4}$ ). That $r=2 M$ is in fact the event horizon is confirmed by a theorem established in Ref. [8]: Let $g_{\alpha \beta}$ be the metric of a perturbed Schwarzschild black hole, let $h_{\alpha \beta}:=g_{\alpha \beta}-g_{\alpha \beta}^{0}$ be the metric perturbation, and let $t^{\alpha}$ be tangent to the null generators of the perturbed horizon; in a coordinate system in which $h_{\alpha \beta} t^{\beta}=0$ on the horizon, the horizon has the same coordinate description in the perturbed and unperturbed spacetimes. In other words, the gauge conditions $h_{\alpha v}=0$ at $r=2 M$ guarantee that the event horizon is located at $r=2 M$ in both spacetimes. Because the metric of Eqs. (71)-(10) satisfies these conditions to order $\mathcal{R}^{-4}$, we conclude that the surface $r=2 M[1+O(4)]$ is the event horizon of the tidally distorted black hole.

The surface gravity $\kappa$ of the perturbed horizon is defined by the statement $t_{; \beta}^{\alpha} t^{\beta}=\kappa t^{\alpha}$, which holds on $r=2 M$ and expresses the fact that the integral curves of $t^{\alpha}$ are null geodesics. A straightforward calculation gives

$$
\kappa=\frac{1}{4 M}\left[1+\frac{16}{3} M^{3} \dot{\mathcal{E}}_{a b}(v) \Omega^{a} \Omega^{b}+O(4)\right] .
$$

The expansion of the congruence of null generators is calculated as $\Theta=\left(g^{\alpha \beta}+t^{\alpha} l^{\beta}+l^{\alpha} t^{\beta}\right) t_{\alpha ; \beta}$, where $l_{\alpha}:=-\partial_{\alpha} v$ is the other null normal to the event horizon. A straightforward calculation reveals that $\Theta=O\left(M^{4} / \mathcal{R}^{5}\right)$. This result implies that within the accuracy of the calculation, each cross section $v=$ constant of the event horizon is in fact an apparent horizon.

The slow growth of the event horizon is not revealed by a direct examination of the horizon's geometry. It can, however, be calculated using the techniques introduced in Ref. 8]. Such a calculation reveals that the averaged rate at which the black hole increases its mass because of tidal heating is given by

$$
\begin{aligned}
\left\langle\frac{d M}{d v}\right\rangle= & \frac{16}{45} M^{6}\left\langle\dot{\mathcal{E}}_{a b} \dot{\mathcal{E}}^{a b}+\dot{\mathcal{B}}_{a b} \dot{\mathcal{B}}^{a b}\right\rangle \\
& +\frac{16}{4725} M^{8}\left\langle\dot{\mathcal{E}}_{a b c} \dot{\mathcal{E}}^{a b c}+\frac{16}{9} \dot{\mathcal{B}}_{a b c} \dot{\mathcal{B}}^{a b c}\right\rangle
\end{aligned}
$$

with a remainder of order $(M / \mathcal{R})^{9}$. The rate at which the horizon's surface area grows is then $\langle d A / d v\rangle=$ $(8 \pi / \kappa)\langle d M / d v\rangle$. The tidal interaction also transfers angular momentum to the black hole; the averaged rate of change of its angular-momentum vector is given by

$$
\begin{aligned}
\left\langle\frac{d J^{a}}{d v}\right\rangle & =-\frac{32}{45} M^{6} \varepsilon^{a}{ }_{b c}\left\langle\dot{\mathcal{E}}^{b}{ }_{d} \mathcal{E}^{c d}+\dot{\mathcal{B}}^{b}{ }_{d} \mathcal{B}^{c d}\right\rangle \\
& -\frac{16}{1575} M^{8} \varepsilon^{a}{ }_{b c}\left\langle\dot{\mathcal{E}}^{b}{ }_{d e} \mathcal{E}^{c d e}+\frac{16}{9} \dot{\mathcal{B}}^{b}{ }_{d e} \mathcal{B}^{c d e}\right\rangle
\end{aligned}
$$

with a remainder of order $M^{9} / \mathcal{R}^{8}$.

Conclusion. The metric of an arbitrary vacuum spacetime was expressed in a light-cone coordinate system centered on a geodesic world line; the metric was expanded to third order in powers of $r / \mathcal{R}$, which is assumed to be small in the coordinate domain. This provided asymptotic conditions on the metric of a tidally distorted, nonrotating black hole, which was obtained by integrating the equations of black-hole perturbation theory to order $\mathcal{R}^{-3}$. The coordinates allow an easy identification of the event horizon, and the effects of the tidal interaction on its physical properties were explored. This work was supported by the Natural Sciences and Engineering Research Council of Canada.

[1] G. Cook, Living Rev. Relativity 3 (2000), 5. [Online article]: cited on February 7, 2008, http://www.livingreviews.org/lrr-2000-5

[2] P.R. Brady, J.D.E. Creighton, and K.S. Thorne, Phys. Rev. D 58, 061501(R) (1998); W. Tichy, B. Brugmann, M. Campanelli, and P. Diener, Phys. Rev. D 67, 064008 (2003); L. Blanchet, Phys. Rev. D 68, 084002 (2003); T. Mora and C.M. Will, Phys. Rev. D 69, 104021 (2004).

[3] K. Alvi, Phys. Rev. D 61, 124013 (2000); 67, 104006 (2003); see also N. Yunes, W. Tichy, B.J. Owen, and B. Brugmann, gr-qc/0503011

[4] K.S. Thorne and J.B. Hartle, Phys. Rev. D 31, 1815 (1985)

[5] P.D. D'Eath, Black holes: Gravitational interactions (Clarendon, Oxford, England, 1996).

[6] S. Detweiler, Phys. Rev. Lett. 86, 1931 (2001).

[7] E. Poisson, Phys. Rev. D 69, 084007 (2004).

[8] E. Poisson, Phys. Rev. D 70, 084044 (2004). 